\title{
Closed kinetic chain (linear) isokinetic testing: Relationships to functional testing
}

\author{
Robert C. Manske a,b,^, Barbara S. Smith ${ }^{\mathrm{a}}$, Michael E. Rogers ${ }^{\mathrm{c}}$ and Frank B. Wyatt ${ }^{\mathrm{d}}$ \\ ${ }^{a}$ Department of Physical Therapy, Wichita State University, Wichita, KS 67260, USA \\ ${ }^{b}$ Via Christi Sports and Orthopedic Physical Therapy, Wichita, KS 67212, USA \\ ${ }^{\mathrm{C}}$ Department of Kinesiology and Sport Studies, Wichita State University, Wichita, KS 67260, USA \\ ${ }^{\mathrm{d}}$ Department of Health, Human Performance, and Recreation, Baylor University, Waco, TX 76798, USA
}

\begin{abstract}
Study design: A correlation study to determine the relationship of the results of linear isokinetic testing and various functional tests.

Objectives: To determine the relationship of scores on a linear isokinetic test to scores on a variety of lower extremity functional tests.

Background: Both isokinetic testing and functional tests are commonly used during the rehabilitation of individuals with sports and orthopedic injuries. Limited information exists regarding the relationships of scores on a linear isokinetic test and scores on functional tests.

Methods and measures: Twenty-nine healthy subjects performed a velocity spectrum linear isokinetic test consisting of 3 sets in a bilateral reciprocal mode, followed by three sets in a bilateral coupled mode. Testing velocities were $25.4,50.8$ and $76.2 \mathrm{~cm}$ per second. Six maximal volitional linear isokinetic repetitions were performed at each testing velocity. Following linear isokinetic testing, subjects performed 5 functional tests: bilateral leg vertical jump, unilateral leg vertical jump, double leg jump for distance, single-leg hop for distance, and single-leg timed 6-meter hop. Functional testing entailed 3 maximal volitional attempts of the 5 procedures for both the dominant and non-dominant lower extremity. The mean of the 3 attempts was used for calculations. Means and standard deviations of the functional test scores and isokinetic test scores were determined using standard statistical procedures. Pearson product moment correlation coefficients were used to determine the relationship between the mean scores on the linear isokinetic test scores and functional tests for both the dominant and non-dominant limbs.

Results: Twenty-three out of 36 correlation coefficients were significant at the $p<0.01$ level, while 12 out of 36 were significant at the $p<0.05$ level. Only 1 out of 36 was non-significant when comparing bilateral reciprocal scores to single-leg functional test scores. The bilateral reciprocal correlation coefficients ranged from -0.36 to 0.65 . Six out of 12 bilateral coupled correlation coefficients were significant at $p<0.01$, while 3 out of 12 were significant at the $p<0.05$ level and only 3 were considered non-significant. The bilateral coupled correlation coefficients ranged from 0.11 to 0.64 .

Conclusions: Results from this study indicate a significant relationship exists between the results of linear isokinetic testing and various functional tests. Despite the significant relationship, the majority of Pearson Product moment correlation coefficients were in the low to moderate range. Therefore, the use of either a linear isokinetic test or a functional test in isolation to determine strength and performance is strongly discouraged. Linear isokinetic testing should be used for testing lower extremity strength, while functional tests should be used to determine performance levels. The authors recommend utilizing both testing methods since strength does not always correlate strongly with physical performance.
\end{abstract}

Keywords: Muscular strength, performance tests, isokinetics

*Address for correspondence: Robert C. Manske, MPT, MEd, SCS, ATC, CSCS, Assistant Professor, Department of Physical Therapy, Wichita State University, 1845 North Faimount, Wi- chita, KS 67260-0043, USA. Fax: +1 316978 3025; E-mail: robert.manske@wichita.edu. 



\section{Introduction}

More stringent demands are being placed on sports and orthopedic clinicians to document functional outcomes. Clinicians must continue to find measurements that are objective, and reliable when evaluating these outcomes. Due to reimbursement issues and shorter coverage for rehabilitation, the need to accurately and effectively assess knee function during initial evaluation and upon subsequent re-examination has become paramount. Traditionally, the evaluation performed following a lower extremity injury has focused on physical characteristics such as measurements of joint effusion, knee ligament laxity, range of motion, muscle strength, and endurance $[25,27,28]$.

Isokinetic dynamometry has become a standard tool for evaluation of and follow-up testing of muscular strength of the lower extremity due to the benefits of range of motion control, accommodating resistance, varying movement velocity, and reliability $[2,6,12,20,30]$. One of the newer modes of isokinetic dynamometers is the Lido Linea Closed Kinetic Chain Isokinetic Dynamometer (Loredan Biomechanical, West Sacramento, CA). This dynamometer has been developed at a time when clinicians have come to rely on closed kinetic chain exercises as a key component in sports and orthopedic rehabilitation. For purposes of this study, closed kinetic chain isokinetic testing will be operationally defined as utilizing a linear isokinetic pattern, while open kinetic chain isokinetic testing will be operationally defined as utilizing an angular pattern.

Until recently, most isokinetic systems utilized an angular pattern of testing in which the distal portion of the extremity tested was free to move. Previous isokinetic studies that have investigated linear strength parameters used a modified dynamometer that provided resistance to a movement through an angular arc of motion similar to the open chain methods $[5,17]$. With newer isokinetic devices, measures of strength and power can be obtained in a linear pattern with the distal extremity fixed to the apparatus $[6,14]$. In addition to the Lido Linea, the Closed Kinetic Chain Rider (Mettler Electronics, Anaheim, CA), and the Cybex Liftask (Cybex Division of Lumex, Inc., Ronkonkoma, NY) are several other examples of isokinetic devices that utilize a linear pattern for strength assessment. To date, only 2 studies have been performed examining the relationships between results of linear isokinetic testing and functional tests $[13,18]$.
Another common means of assessing lower extremity strength is through the performance of functional tests. Functional tests are activities that can be objectively measured that closely simulate sports or recreational activity. In addition to muscular strength these functional tests also assess neuromuscular control, speed of muscle contraction, lower extremity power, joint function and joint range of motion. These tests are numerous and a partial lists includes single-leg tests such as the one-legged hop test for distance $[3,8,9,19]$, the timed one-legged hop test over a distance of 6 meters [3], the one-legged vertical jump [3], the crossover triple hop [19], and the leap [3,11]. In addition to these tests, two-legged tests are available: the figureof-eight-test [29] the stair-running test [29], the carioca test [15], the shuttle run [16], the semicircular test and the vertical jump test [15].

Many studies have assessed the relationship between results of isokinetic tests and functional tests. Ironically, each of these studies compared an angular pattern of isokinetic testing with closed kinetic chain functional test $[1,3,4,7,15,21,23,26,31]$. The nature of the two different testing procedures, (i.e., using functional tests that are closed kinetic chained and isokinetic tests that are angular) would suggest that relationships may be low. Only a few studies have assessed relationships between linear isokinetic testing and functional performance testing [13,18].

Negrete and Brophy [18] compared results of angular and linear isokinetic testing with several functional tests. Using a group of 60 volunteers, results of isokinetic knee extension, leg press, and single-leg squat strength were correlated with functional test scores of single-leg hop, vertical jump and a speed/agility test. Isokinetic leg press strength evaluated at $76.2 \mathrm{~cm} / \mathrm{s}$ demonstrated moderate to high correlations $(r=0.67$ to 0.76 ) with 2 unilateral performance tests: the singleleg hop for distance and the single-leg vertical jump.

More recently, Kovaleski et al. [13] utilized both angular and linear isokinetic testing to determine their relationships to functional performance. In their study, 30 uninjured men and women were tested to determine relationships between low-speed isokinetic testing and performance on functional tasks such as jumping, hopping, and speed agility. Neither lower-limb linear nor angular isokinetic strength measurements correlated highly ( $r=0.26$ to 0.62 ) with the functional test results.

Therefore, the primary purpose of this study was to assess the relationship between linear concentric isokinetic test scores on the Lido Linea and scores on a series 
of closed kinetic chain functional tests. The research hypothesis guiding this study was that linear concentric isokinetic testing scores obtained on the Lido Linea would have a positive association with jumping and hoping closed kinetic chain lower extremity functional test scores, and have a negative association with results of the 6-meter single-leg timed hop.

\section{Methods}

A sample of 29 healthy participants aged 19-33 years $($ mean $=26.7+/-5.2$ ) with no history of prior knee surgery or recent acute knee injury were recruited through a university physical therapy department and through a local sports and orthopedic physical therapy outpatient clinic. Sixteen of the participants were men, while 13 were female. Means, standard deviations and range of participants weight and height can be seen in Table 1. Participants were evaluated for lower extremity pathology through a self-reported health history questionnaire and through a physical examination by the principal author to assess any instability in the knees or ankles. A manual physical examination including the Lachman's Test, posterior drawer, varus and valgus stress to the knee were all performed prior to testing. In addition, ankle joint anterior drawer, and varus/valgus stress testing were also performed to rule out instability at the knee or ankle as a possible cause of low correlations during our study. Any participant with a recent knee or ankle injury (within past 9 months), past surgical procedure(s) to one or both knees, ligamentous instability, or loss of balance found on examination was excluded from the study. Participant's balance was assessed via a single leg stance test. This test had to be held for a minimum of 30 seconds without loss of balance for the participant to be included in out study. No participant demonstrated a loss of balance, which would have excluded them from the study. A health history questionnaire determined if the participants ever had an upper extremity injury that would hinder their ability to reach during the vertical jumping. No subjects reported a prior history of upper or lower extremity injury or surgery. All 29 subjects started and finished our study. Leg dominance was determined by asking participants with which leg they would be most comfortable kicking a ball. Prior to testing each participant received an explanation of the risks, benefits, procedures and signed a consent form approved by the university's and medical center's Institutional Review Boards.
Table 1

Mean, standard deviation, and range of subjects' ( $n=29)$ age, height, and weight

\begin{tabular}{lrrc}
\hline & Mean & SD & Range \\
\hline Age (years) & 26.7 & 5.2 & $19-37$ \\
Height $(\mathrm{cm})$ & 175.3 & 10.4 & $157.5-193.0$ \\
Weight $(\mathrm{kg})$ & 75.1 & 14.8 & $47.3-106.2$ \\
\hline
\end{tabular}

\subsection{Procedures}

Participants performed the tests beginning with a brief warm-up followed by isokinetic testing and finally the performance of four functional tests, followed by a brief cool-down. The surface on which the functional testing was performed was a low-pile carpet glued over a concrete floor. Functional tests were chosen due to their prevalence in the orthopedic and sports medicine literature. All testing was performed in a non-randomized order beginning with isokinetic tests first, followed by the functional tests. This was done purposefully to more closely simulate actual rehabilitation clinical conditions in which functional testing is usually preceded with isokinetic testing. All functional tests were performed in a systematic manner beginning with bilateral leg tests progressing to unilateral leg testing. Functional tests progressed from those with the least stress applied to the lower extremities, e.g., bilateral vertical jump, to those with the most stress applied to the lower extremities, e.g., 6-m timed singleleg hop. Three practicing sports and orthopedic physical therapists, all with a minimum of 6 years of clinical experience, performed all testing. All of the testing procedures were performed on the same day. The entire testing session lasted approximately 40 minutes for each participant. One therapist performed all of the isokinetic tests, while the other 2 therapists performed the functional tests.

\subsection{Instrumentation}

The Lido Linea Closed Kinetic Chain Isokinetic Dynamometer was used to measure strength and power performance during concentric linear isokinetic leg press testing in both bilateral reciprocal pattern and bilateral coupled pattern. A bilateral reciprocal repetition on the Linea is defined as a complete leg press stroke from the flexed knee position to the extended knee position for each limb in direct succession (each pedal moved independent or unlocked). A bilateral coupled leg press on the Linea is defined as a complete leg press stroke from the flexed position to the extended position with both extremities in unison (pedals moved 
together or locked). The Lido Linea has been shown to be reliable [6]. Measurements of peak force were recorded for each limb by the system's software. Static calibration was performed per instruction manual and has been previously described [6].

Participants performed a standardized warm-up prior to each testing session. The standardized warm-up consisted of five minutes of cardiovascular and musculoskeletal exercise on a stationary bicycle at a selfselected, submaximal intensity.

Participants were positioned semi recumbent in the Linea's leg press chair and stabilized by a lap belt. Participants were placed in the Lido Linea with their knees fully extended, and with their hips in approximately $70^{\circ}$ of flexion. This position was standardized to ensure that the length of the trunk, thigh and legs could not be a cause of movement error during testing. A linear range of motion was established so that the knees moved from approximately $90^{\circ}$ of knee flexion to $5^{\circ}$ of flexion during the complete leg press stroke. A popliteal pad was placed behind the knees to prevent knee hyperextension. Participants were allowed to grasp the system's handgrips during testing.

Prior to each test, participants performed an linear isokinetic warm-up of three gradient, submaximal repetitions plus one maximal repetition at each test velocity. The submaximal warm-up repetitions progressed from $25-50-75 \%$ of volitional effort. Participants performed the bilateral coupled test followed by the bilateral reciprocal leg press test motion.

Testing consisted of a velocity spectrum of linear isokinetic leg press exercises performed in the concentric mode at slow $(25.4 \mathrm{~cm} / \mathrm{s})$, medium $(50.8 \mathrm{~cm} / \mathrm{s})$, and fast $(76.2 \mathrm{~cm} / \mathrm{s})$ velocities. For each test velocity, six successive maximal volitional concentric isokinetic repetitions were performed. An isokinetic test velocity on the Lido Linea refers to a constant change in the linear shaft over time. Strong verbal encouragement was provided in a consistent manner to elicit maximal effort from the participant. Participants were provided visual feedback of the performance. A one-minute rest period separated testing at each velocity. The testing sequence progressed from slow to medium to fast velocities, which is common in clinical isokinetic strength testing.

At the conclusion of Lido Linea isokinetic testing, participants performed light lower extremity stretching at their discretion. These stretches generally consisted of quadriceps and hamstring stretching exercises for up to 3 minutes. Isokinetic testing preceded functional testing.
Following the warm-up, isokinetic strength tests, and stretching, each participant performed the following functional tests: the bilateral vertical jump test, singleleg vertical jump, 2-legged jump test, single-leg hop test, and the timed 6-m single-leg hop test. These tests were chosen for this study due to their ease of use in a clinical setting and the ability to perform them in our clinic. Three warm-up trials for each functional test were allowed for familiarity with the actions required and to ensure competence and understanding with instructions for the testing procedures. Participants performed 3 attempts of each test and were asked to give maximal effort for each attempt. A one-minute rest period was allowed between tests. Upper extremity movement was restricted as much as possible when appropriate. At completion of functional testing, participants performed a cool down by riding a stationary bicycle and or stretching.

\subsection{Vertical jump test}

Sargent [22] and Semenick [24] previously described the standing vertical jump test. It is generally accepted that using traditional chalk or tape methods of assessing vertical jump can generate a large amount of error, so the Vertec jump apparatus (Sports Imports, Inc., Columbus, Ohio) was used. A standing baseline reach measurement was initially performed with participants standing flat footed and reaching as high as possible with both the right, then the left upper extremity. Participants then jumped as high as possible with both legs, the dominant leg, and with the non-dominant leg. Three trials were completed with both legs together and with each single leg. Participants were asked to jump as high as possible and to reach with the opposite upper extremity to displace a movable plastic vane of 0.5 -inch increments. Counter movements with the upper extremities were utilized during this test because reaching is a part of the testing procedure. The standing baseline reach was subtracted from the total vertical jump score to obtain the distance jumped.

The 2-legged jump test and the single-leg hop test were performed in a similar fashion to each other. The Standing Long Jump Mat (SBP, Toronto, Canada) composed of a durable rubber material was used for measuring jumps and hops. This mat has a standardized starting position and is able to measure jumps of 50 to $320 \mathrm{~cm}$. Participants were asked to stand with the front of their shoes at the starting mak. Participants stood with the front of their shoes behind the starting mark with hands on hips and knees bent. Participants 
jumped or hopped (2-legged or single-leg hop) as far horizontally as possible, landing with bent knee(s) to help decrease risk of injury. During performance of the 2-legged jump for distance the participant jump from both feet and landed on both feet. During the singleleg hop for distance, the participant hopped from a single leg and landed on that same leg from which they hopped off of. The spot where the heel of the more posterior shoe landed was marked and used as jump distance. To qualify for a legitimate jump, participants had to land without falling down or taking a step. After landing, this position had to be held for at least $2 \mathrm{sec}-$ onds. A failed jump included loss of balance, touching floor with the contralateral lower extremity (during single-leg hop), the upper extremities, or an additional hop after landing. The distance of 3 maximal attempts for each test was recorded.

When testing the single-leg hop, participants hopped using the non-dominant leg first followed by the dominant leg. Once again, as with the 2-legged jump test, participants hands were held at the hips to decrease gaining momentum with the arms. Participants bent their knees and hopped as far horizontally as possible, this time landing on the same extremity. The distance from the starting mark to the heel of the landing leg was measured. Participants had to land without losing balance and without relying on the other lower extremity to aid in landing.

Barber et al. [3] have described the single-leg 6-m timed hop. The single-leg timed $6-\mathrm{m}$ hop began at a mark of standard athletic tape placed on the floor. The single-leg timed 6-m hop was performed using large forceful single-leg hopping motions across the floor. A standard manual stopwatch was used to time each trial to the nearest $1 / 100$ second. Three trials were performed for each lower extremity with the nondominant leg tested first, followed by the dominant leg.

\subsection{Data analysis}

Mean and standard deviations for isokinetic scores and scores on functional tests were calculated using standard statistical procedures. Pearson product moment correlation coefficients were used to determine the relationship between the mean scores on the functional tests and the mean scores of semi recumbent leg extension peak force (ft-lb) isokinetic linear strength measurements for the dominant and non-dominant limbs. An alpha level was set at $p \leqslant 0.05$. Statistical analysis was performed using Statistical Package for Social Sciences (SPSS, V. 9.0, Chicago, IL).

\section{Results}

A total of 29 subjects (19-33 years of age) agreed to complete the research study. All 29 subjects started and ended the testing procedures. Table 2 shows mean, standard deviations and ranges of the functional test scores for each variable measured.

Data from the bilateral reciprocal concentric isokinetic peak force scores showed relationships to singleleg functional test scores ranging from -0.36 to 0.65 (Table 3). Negative correlations are due to the two different measurement methods. For isokinetic testing, the relative scores increase numerically as the person obtains a higher score. Thus, a greater peak force generation will result in a greater isokinetic score. In performance of the single-leg 6-m timed hop, the greater amount of time taken to hop 6-m results in a worse or lower score. A subject with a higher or better score is able to perform the test in a shorter time. Therefore, a negative correlation exists.

With the exception of one comparison, all of the functional test scores demonstrated significant correlations ( $p \leqslant 0.05$ ) with each linear testing parameter. The test not showing significance was between the singleleg hop and the bilateral reciprocal mode, dominant leg at $76.2 \mathrm{~cm}$. per second $(p=0.059)$. An interpretation of the coefficients of determination (r2) for these tests indicates that the single-leg hop tests account for only $15 \%$ to $42 \%$ of the variation in the isokinetic measures.

Relationships between bilateral coupled concentric isokinetic peak force scores and double-leg functional test scores demonstrated positive correlation coefficients ranging from 0.11 to 0.64 (Table 4 ). The scores for the 2-legged hop tests account for only $1 \%$ to $41 \%$ of the variation in the isokinetic measures.

\section{Discussion}

No study to date has compared velocity spectrum linear concentric isokinetic testing scores with functional performance tests. The results of this study show low to moderate, yet statistically significant relationships, between strength on a linear concentric isokinetic leg press and the performance of functional tests such as the bilateral vertical jump test, single-leg vertical jump, double-leg jump for distance, single-leg hop test and the timed 6-meter single-leg hop test. Most correlations were significant at the $p \leqslant 0.05$. In addition, most tests demonstrated correlations in the low to moderate levels with $\mathrm{r}$-values in most cases greater than 0.25 . A 
Table 2

Mean, standard deviation, and range of functional test scores

\begin{tabular}{lrcc}
\hline & Mean & SD & Range \\
\hline 2-legged jump B (cm) & 162.1 & 26.7 & $110.5-224.0$ \\
Single-leg hop DL (cm) & 126.5 & 21.6 & $88.1-185.4$ \\
Single-leg hop NDL $(\mathrm{cm})$ & 127.8 & 28.2 & $86.4-188.0$ \\
Vertical jump - B (cm) & 42.7 & 10.8 & $27.2-64.8$ \\
Vertical jump - DL (cm) & 27.4 & 8.4 & $17.0-44.5$ \\
Vertical jump - NDL (cm) & 31.5 & 10.4 & $17.8-57.7$ \\
Single-leg timed 6 m hop DL (sec) & 2.1 & 0.36 & $1.7-2.9$ \\
Single-leg timed 6 m hop NDL (sec) & 2.1 & 0.42 & $1.49-3.33$ \\
\hline
\end{tabular}

$\mathrm{B}=$ Bilateral $; \mathrm{DL}=$ Dominant Lower Extremity; NDL $=$ Non-dominant Lower Extremity.

Table 3

Correlation coefficients for bilateral reciprocal concentric isokinetic peak force and single-leg functional test scores

\begin{tabular}{lcccccc}
\hline & BR-DL10 & BR-NDL10 & BR-DL20 & BR-NDL20 & BR-DL30 & \multicolumn{2}{c}{ BR-NDL30 } \\
& $\mathrm{r}$ & $\mathrm{r}$ & $\mathrm{r}$ & $\mathrm{r}$ & $\mathrm{r}$ & $\mathrm{r}$ \\
\hline SLHD & $0.51^{* *}$ & $0.51^{*}$ & $0.45^{*}$ & $0.55^{*}$ & $0.44^{*}$ & $0.49^{*}$ \\
SLHN & $0.49^{* *}$ & $0.55^{*}$ & $0.48^{*}$ & $0.65^{*}$ & $0.45^{*}$ & $0.57^{* *}$ \\
VJD & $0.39^{*}$ & $0.38^{*}$ & $0.44^{*}$ & $0.49^{*}$ & $0.47^{*}$ & $0.52^{* *}$ \\
VJN & $0.40^{*}$ & $0.39^{*}$ & $0.45^{*}$ & $0.55^{*}$ & $0.45^{*}$ & $0.54^{\cdots}$ \\
STHD & $-0.51^{* *}$ & $-0.48^{*}$ & $-0.45^{* *}$ & $-0.53^{*}$ & $-0.46^{*}$ & $0.50^{*}$ \\
STHN & $-0.48^{* *}$ & $-0.52^{*}$ & $-0.39^{*}$ & $-0.58^{* *}$ & -0.36 & $-0.50^{* *}$ \\
\hline
\end{tabular}

Note: $"{ }^{\prime} p<0.01$ level (2-tailed); ${ }^{*} p<0.05$ level (2-tailed). BR-DL10 = Bilateral reciprocal dominant leg $10 \% / \mathrm{sec}$.

BR-NDL10 = Bilateral reciprocal non-dominant leg $10 / \mathrm{sec}$. BR-DL20 = Bilateral reciprocal dominant leg $20 \% / \mathrm{sec}$.

BR-NDL20 = Bilateral reciprocal non-dominant leg $20 \% / \mathrm{sec}$. BR-DL $30=$ Bilateral reciprocal dominant leg $30 \% / \mathrm{sec}$.

BR-NDL 30 = Bilateral reciprocal non-dominant leg $30^{\circ} / \mathrm{sec}$.

SLHD $=$ Single-leg hop for distance dominant leg.

SLHN $=$ Single-leg hop for distance non-dominant leg.

VJD $=$ Vertical jump unilateral dominant leg.

VJN $=$ Vertical jump unilateral non-dominant leg.

STHD $=$ Single-leg timed $6 \mathrm{~m}$ hop dominant leg.

$\mathrm{STHN}=$ Single-leg timed $6 \mathrm{~m}$ hop non-dominant leg.

correlation coefficient of 0.90 to 1.00 is interpreted as a very high correlation, a 0.70 to 0.89 is interpreted as a high correlation, 0.50 to 0.69 is considered a moderate correlation, while 0.26 to 0.49 is considered a low correlation [10].

Negrete and Brophy [18] found significant correlations between functional test scores and linear concentric isokinetic test scores. The single-leg hop and the bilateral reciprocal leg press motion were correlated at 0.73 , while the isokinetic single-leg squat and the single-leg hop were correlated at 0.73 , with each significant at the $p<0.001$ level. The correlation between the single-leg vertical jump and the bilateral leg press was 0.58 , while with the isokinetic single-leg squat correlated at 0.57 , again, both significant at the $p<0.001$ level.

One potential difference between results of Negrete and Brophy [18] and those of the present study is the position that participants were placed in the isokinetic machine. The present study used a semi recumbent position, similar to that used by Davies and Heiderscheit [6] during their study to determine reliability of the Lido Linea. The Negrete and Brophy [18] study used a true supine position. Theoretically this supine position could have altered the biomechanics of the hip and knee and ankle enough to more truly simulate functional testing. The full supine position would place the hip and knee extensors in better length-tension relationships to allow greater torque production. The present study, with subjects placed in a semi recumbent position, may not have allowed full torque production of the hip and knee extensor muscles or the ankle plantar flexor muscles. This could have slightly reduced the coefficients seen in this study.

The low to moderate correlations found in this and the study by Negrete and Brophy [18] could have im- 
Table 4

Correlation coefficients for bilateral coupled concentric isokinetic peak force and two-legged functional test scores

\begin{tabular}{|c|c|c|c|c|c|c|}
\hline & $\begin{array}{c}\text { BC-DL10 } \\
\mathrm{r}\end{array}$ & $\begin{array}{c}\text { BC-NDL10 } \\
\mathrm{r}\end{array}$ & $\begin{array}{c}\text { BC-DL20 } \\
\mathrm{r}\end{array}$ & $\begin{array}{c}\text { BC-NDL20 } \\
\mathrm{r}\end{array}$ & $\begin{array}{c}\text { BC-D30 } \\
\mathrm{r}\end{array}$ & $\begin{array}{c}\text { BC-NDL } 30 \\
\mathrm{r}\end{array}$ \\
\hline TLJ & 0.11 & $0.46^{*}$ & $0.38^{*}$ & $0.59^{* *}$ & $0.43^{*}$ & $0.59^{* *}$ \\
\hline VJB & 0.16 & 0.47 & $0.48^{*}$ & $0.64^{\cdots}$ & $0.53^{*}$ & $0.61^{*}$ \\
\hline \multicolumn{7}{|c|}{ 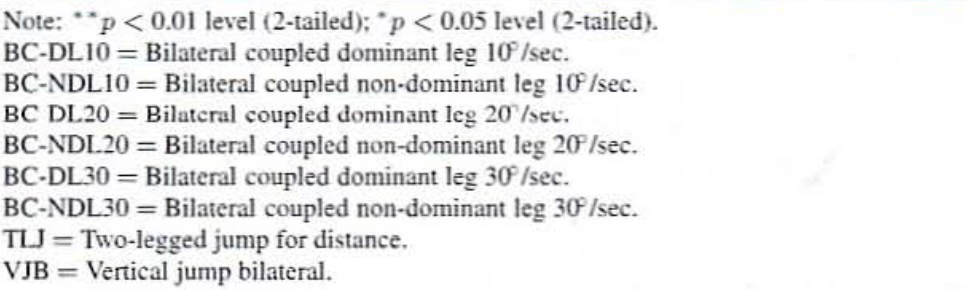 } \\
\hline
\end{tabular}

portant clinical applications. Although generalizations to a injured population are difficult to make from our study of normal participants, we feel that the present study demonstrates that one single test, whether it be an isokinetic test or a functional test, may not be sufficient to entirely determine a participants strength and ultimate functional level or safe ability to participate in sporting activities. Although testing was done in a linear fashion, low to moderate correlations suggest that the Lido Linea still does not totally emulate a functional test. The linear motion of the Lido Linea functions at a preset velocity with a constrained range of motion during knee extension and flexion. Therefore, varus, valgus, and rotary forces at the hip, knee and ankle are non-existent. This is in direct contrast to a functional test in which the participant has to deal with gravity, balance, proprioception, kinesthesia, varus, valgus and rotary forces at the knee, hip, and ankle. In addition, velocity, range of motion, and eccentric deceleration forces are not limited by functional testing. Therefore, even though each method i.e., isokinetic and functional, is performed in a closed chain manner, they are quite different. The low to moderate correlations between the functional tests and linear isokinetic testing may suggest that these functional tests may be influenced by other factors such as trunk strength, upper extremity strength, balance and proprioception. Therefore, we feel that linear isokinetic testing should be utilized to determine only the strength or muscle performance of the entire lower extremity chain. During linear testing it must be emphasized that a decrease in strength of any single joint along the kinetic chain may be compensated for by another joint in that chain. For instance, weakness of the quadriceps muscle may be hidden in the linear isokinetic test if the participants' hip and ankle extensors have increased in strength as a compensatory pattern. For testing muscular strength of a single joint, an angular method of isokinetic testing would be most appropriate. Furthermore, functional testing should be used to assess true "function" of the lower extremity. Again it must be stressed that "function" does not always correlate strongly with "strength". Our recommendation is to utilize both forms of isokinetic testing if available. Linear isokinetic testing will give the clinician true objective measures of the cumulative strength of the entire kinetic chain musculature, while angular isokinetic testing can assess muscular strength at each individual joint. If the participant being testing demonstrates favorable scores on linear isokinetic tests, functional testing could be the next progression. Functional testing can also give objective measures regarding ones physical performance level.

Correlations between linear isokinetic testing with the non-dominant leg and functional testing are higher than correlations using scores from the dominant leg. This study used the standard technique of utilizing the leg that one would use to kick a ball to determine dominance. The finding that the non-dominant leg demonstrated higher correlations between linear isokinetic strength scores and functional test scores than the dominant leg may be important to the field of rehabilitation and physical therapy. Should dominance be determined with respect to which mode of strength testing is utilized? The standard method of determining dominance by having the participants kick a ball may be very appropriate for angular testing, since kicking a ball is an open chain activity. But the present study used a linear isokinetic testing apparatus, and functional testing is a closed chain activity. When using the standard method of determining dominance, the non-dominant leg is the leg most used in a closed kinetic chain fashion during kicking a ball. This could explain why, when testing in a linear manner, the scores on the non-dominant, i.e., closed chain leg, demonstrated higher correlations mentioned above. 


\section{Conclusions}

The single-leg and 2-legged functional tests used in the present study are both clinical tests that provide qualitative and quantitative data regarding the physical function of the knee. Furthermore, each of these tests can be performed easily in a clinical setting without expensive isokinetic testing devices such as the Lido Linea. Despite this, the present study showed that the correlations between these tests and closed kinetic chain isokinetic test scores are in the low to moderate range. Neither functional testing nor isokinetic tests should be used in isolation when attempting to determine both muscle performance and physical function of the knee and lower extremity.

\section{Acknowledgments}

The authors gratefully than Mark Needham, Julie Oler-Manske, and Kim Yearout for their assistance during this project.

\section{References}

[1] M.A. Anderson, J.H. Gieck. D. Perrin, A. Weltman, R. Rutt and C. Denegar, The relationship among isometric, isotonic, and isokinetic concentric and eccentric quadriceps and hamstring force and three components of athletic performance, $J$ Orthop Sports Phvs Ther 3 (1991), 114-120.

[2] J. Barbee and D. Landis, Reliability of Cybex computer measures, Phys Ther 5 (1984), 737.

[3] S.D. Barber, F.R. Noyes, R.E. Mangine, J.W. McClosky and W. Hartman, Quantitative assessment of functional limitations in normal and anterior cruciate ligament-deficient knees, Clin Orthop 255 (1990), 204-214.

[4] J.R. Blackburn and M.C. Morrissey. The relationship between open an closed kinetic chain strength of the lower limb and jumping performance, J Orthop Sports Phys Ther 6 (1998), $430-435$.

[5] M.L. Cordova, C.D. Ingersoll, J.E. Korvaleski and K.L. Knight, A comparison of isokinetic and isotonic predictions of a functional task, J Athl Train 30 (1995), 319-322.

[6] G.J. Davies and B.C. Heiderscheit, Reliability of the Lido Linea closed kinetic chain isokinetic dynamometer, J Orthop Sports Phys Ther 25 (1997), 133-136.

[7] A. Delitto, J.J. Irgang, C.D. Harner, F. Fu and S. Nessi, Relationship of isokinetic quadriceps peak torque and work to onelegged hip and vertical jump in ACL reconstructed subjects (abstract), J Orthop Sports Phys Ther 6 (1993), S85.

[8] H.B. Greenberger and M.V. Paterno, The test-retest reliability of a one-legged hop for distance in healthy young adults (abstract), J Orthop Sports Phys Ther 1 (1994), 62.

[9] H.B. Greenberger and M.V. Paterno, Comparison of an isokinetic strength test and a functional perfonanace test in the assessment of lower extremity function (abstract), $J$ Orthop Sports Phys Ther 1 (1994), 61.
[10] D.E. Hinkle, W. Wiersma and S.G. Jurs, Applied Statistics for the Behavioral Sciences, Boston, MA: Houghton Mifflin Company, 1998.

[11] P.M. Juris, E.M. Phillips, C. Dalpe, C. Edwards, R.S. Gotlin and D.J. Kane, A dynamic test of lower extremity function following anterior cruciate ligament reconstruction and rehabilitation, J Orthop Sports Phys Ther 4 (1997), 184-191.

[12] P. Kannus, Isokinetic evaluation of muscle performance: implications for muscle testing and rehabilitation. Int J Sports Med 15 (1994), s11-s18.

[13] J.E. Kovaleski, R.J. Heitman, D.P.S. Andrew, L.R. Gurchiek and A. Pearsall, Relationship bẹtween closed-linear-kinetic and open-kinetic-chain isokinetic strength and lower extremity functional performance, J Sports Rehabil 10 (2001), 196-204.

[14] J.E. Kovaleski, R.J. Heitman, L.R. Gurchiek, J.W. Erdmann and T.L. Trundle, Reliability and effects of leg dominance of lower extremity isokinetic force and work using the closed chain rider system, J Sports Rehabil 6 (1997), 319-326.

[15] S.M. Lephart, D.H. Perrin, F. Fu, J.H. Gieck, F.C. McCue and J.J.Irrgang, Relationships between selected physical characteristics and functional capacity in the anterior cruciate ligament-insufficient athlete, $J$ Orthop Sports Phys Ther 4 (1992), 174-181.

[16] S.M. Lephart, D.H. Perrin, F. Fu and K. Minger, Functional performance tests for the anterior cruciate ligament insufficient athlete, J Athl Train 26 (1991), 44-50.

[17] D. Levine and A. Klein, Reliability of isokinetic concentric closed kinematic chain testing of the hip and knee extensors. Isokinetic Exerc Sci 1 (1991), 146-152.

[18] R. Negrete and J. Brophy. The relationship between isokinetic open and closed chain lower extremity strength and functional performance, J Sports Rehabil 9 (2000), 46-61.

[19] F.R. Noyes, S.D. Barber and R.E. Mangine, Abnormal lower limb symmetry determined by functional hop tests after anterior cruciate ligament rupture, Am J Sports Med 5 (1991), 513-518.

[20] D.H. Perrin, Reliability of isokinetic measures, $J$ Athl Train 4 (1994), 212-217.

[21] R. Petschnig, R. Baron and M. Albrecht. The relationship between isokinetic quadriceps strength test and hop tests for distance and one-legged vertical jump test following anterior cruciate ligament reconstruction, $J$ Orthop Sports Phys Ther 1 (1998), 23-31.

[22] D.A. Sargeant, The physical test of a man, Am Phys Ed Rev 26 (1921), 188-194.

[23] I. Sekiya, T. Muneta, T. Ogiuchi, K. Yagishita and K. Yamamoto, Significance of the single-legged hop test to the anterior cruciate ligament-reconstructed knee in relation to muscle strength and anterior laxity, Am J Sports Med 26 (1998). $384-388$.

[24] D. Seminick, The vertical jump, J Strength Cond Res 12 (1990), 68-69.

[25] J.L. Setto, A.S. Orofino, M.C. Morrissey, J.M. Medeiros and W.J. Mason, Assessment of quadriceps/hamstring strength. knee ligament stability, functional and sports activity levels five years after anterior cruciate ligament reconstruction, $J$ Orthop Sports Phys Ther 2 (1988), 170-180.

[26] S.W. Shaffer, E.D. Payne, L.R. Gabbard and J.S. Halle, Relationship between isokinetic and functional tests of the quadriceps (abstract), J Orthop Sports Phys Ther 1 (1994), 55.

[27] D.K. Shelbourne and D.A. Foulk, Timing of surgery in acute anterior cruciate ligament tears on the return of quadriceps strength after reconstruction using an autogenous patellar tendon graft, Am J Sports Med 6 (1995), 686-689. 
[28] M.F. Sherman, L. Lieber, J.R. Bonamo, L. Podesta and 1. Reiter, The long-term follow-up of primary anterior cruciate ligament repair, Am J Sports Med 3 (1991), 243-255.

[29] Y. Tegner and J. Lysholm, Rating systems in the evaluation of knee ligament injuries, Clin Orthop 198 (1985), 43-49.

[30] K.E. Wilk, R.D. Johnson and B. Levine, Reliability of the Biodex B-2000 isokinetic dynamometer (abstract), Phys Ther 68 (1988), 792.
[31] K.E. Wilk, W.T. Romaniello, S.M. Soscia, C.A. Arrigo and J.A. Andrews, The relationship between subjective knee scores, isokinetic testing, and functional testing in the ACLreconstructed knee, J Orthop Sports Phys Ther 2 (1994), 6073. 